\title{
Reproducible in vivo detection of locus coeruleus pathology in Parkinson's Disease
}

\author{
Kristy S. Hwang ${ }^{1+}$, Jason Langley ${ }^{2+}$, Richa Tripathi ${ }^{3}$, Xiaoping P. Hu ${ }^{2,4}$, and Daniel E. \\ Huddleston ${ }^{5}$ \\ ${ }^{1}$ Department of Neurosciences, University of California San Diego, San Diego, CA, USA \\ ${ }^{2}$ Center for Advanced Neuroimaging, University of California Riverside, Riverside, CA, USA \\ ${ }^{3}$ Department of Neurology, Rockefeller Neuroscience Institute, West Virginia University, Morgantown, \\ WV, USA \\ ${ }^{4}$ Department of Bioengineering, University of California Riverside, Riverside, CA, USA \\ ${ }^{5}$ Department of Neurology, Emory University, Atlanta, GA, USA \\ +These authors contributed equally \\ E-mail: daniel.huddleston 'at' emory.edu
}

\section{Abstract}

Background: Patients with Parkinson's disease undergo a loss of melanized neurons in substantia nigra pars compacta and locus coeruleus. There are very few in vivo studies of locus coeruleus pathology in Parkinson's disease with magnetic resonance imaging. Existing studies have used varying methodologies, and reproducibility has not been established for any approach.

Objectives: We used neuromelanin-sensitive magnetic resonance imaging to examine the reproducibility of locus coeruleus and substantia nigra pars compacta degeneration in discovery and validation cohorts.

Methods: Two cohorts, discovery and validation, recruited from the Emory Movement Disorders Clinic were scanned on two different magnetic resonance imaging scanners. In cohort 1, imaging data from 19 controls and 22 Parkinson's disease patients were acquired with a Siemens Trio 3 Tesla scanner using a 2D gradient echo sequence with magnetization transfer preparation pulse. Cohort 2 consisted of 33 controls and 39 Parkinson's disease patients were scanned on a Siemens Prisma 3 Tesla scanner with a similar imaging protocol. Nigral and locus coeruleus volumes were segmented in both cohorts.

Results: Nigral volume (Cohort 1: $p=0.0148$; Cohort 2: $p=0.0011$ ) and locus coeruleus volume (Cohort 1: $p=0.0412$; Cohort 2: $p=0.0056$ ) were significantly reduced in the Parkinson's disease group as compared to controls in both cohorts.

Interpretation: Substantia nigra pars compacta volume and locus coeruleus volume were significantly reduced in Parkinson's disease compared to controls in both cohorts. This imaging approach robustly detects Parkinson's disease effects on these structures suggesting its use as a potential diagnostic biomarker of Parkinson's disease.

Keywords: neuromelanin, substantia nigra, locus coeruleus, Parkinson's disease

\section{Introduction}

Parkinson's disease (PD) is a heterogeneous neurodegenerative disorder with a variety of motor and nonmotor somptoms that can be clinicallit challenging to th diagnose and manage. By the time of symptom onset and clinical diagnosis, there is an estimated $50-70 \%$ of dopaminergic neuronal loss in the substantia nigra compacta $(\mathrm{SNc}) .^{1-3}$ There are currently no effective neuroprotective interventions to stop PD neurodegeneration. Historically, SNc degeneration is known as the pathological hallmark of 
medRxiv preprint doi: https://doi.org/10.1101/2022.02.23.22271356; this version posted February 24,2022 . The copyright holder for this

preprint (which was not certified by peer review) is the author/funder, who has granted medRxiv a license to display the preprint in perpetuity.

All rights reserved. No reuse allowed without permission.

\begin{tabular}{lccc|ccc}
\hline \hline \multirow{2}{*}{ Group characteristics } & \multicolumn{3}{c|}{ Cohort 1 } & \multicolumn{3}{c}{ Cohort 2 } \\
\cline { 2 - 7 } & Control & PD & $\boldsymbol{P}$ & Control & PD & $\boldsymbol{P}$ \\
\hline Participants & 19 & 22 & - & 33 & 39 & - \\
Age & $71.3 \pm 1.2$ & $60.4 \pm 1.8$ & $<\mathbf{1 0}-\mathbf{3}$ & $63.5 \pm 1.6$ & $63.8 \pm 1.6$ & 0.894 \\
Gender (M:F) & $9: 10$ & $13: 9$ & 0.453 & $11: 22$ & $22: 17$ & 0.50 \\
Education [years] & $18.2 \pm 0.5$ & $16.3 \pm 0.5$ & $\mathbf{0 . 0 1 5}$ & $16.7 \pm 0.4$ & $17.0 \pm 0.6$ & 0.661 \\
Race [\% Caucasian] & $78.9 \%$ & $100 \%$ & $\mathbf{0 . 0 2 3}$ & $90.6 \%$ & $87.2 \%$ & 0.402 \\
Disease Duration [years] & - & $6.8 \pm 0.7$ & - & - & $3.5 \pm 0.6$ & - \\
UPDRS-III & $3.2 \pm 0.6$ & $18.7 \pm 2.3$ & $<\mathbf{1 0}^{-3}$ & $1.8 \pm 1.9$ & $18.9 \pm 1.1$ & $<\mathbf{1 0}^{-3}$ \\
Levodopa Equivalents & - & $768.8 \pm 90.4$ & - & - & $623.3 \pm 70.8$ & - \\
MoCA & $27.5 \pm 0.5$ & $27.1 \pm 0.6$ & 0.566 & $28.0 \pm 0.3$ & $27.8 \pm 0.3$ & 0.621 \\
RBD-SQ & $2.3 \pm 0.4$ & $4.7 \pm 0.6$ & $\mathbf{0 . 0 0 2}$ & $2.7 \pm 0.4$ & $3.7 \pm 0.4$ & 0.087 \\
NMSQ & $3.4 \pm 0.7$ & $10.5 \pm 0.9$ & $<\mathbf{1 0}-\mathbf{3}$ & $3.2 \pm 0.5$ & $6.6 \pm 0.7$ & $\mathbf{0 . 0 0 0 6}$ \\
\hline \hline
\end{tabular}

Table 1. Demographic information for the groups used in this analysis. Data is presented as mean \pm standard error unless noted otherwise. Two-tailed $t$-tests were used for group comparisons of age, education, MoCA, RBD-SQ, and NMSQ from which $\mathrm{p}$ values are shown. UPDRS-III was measured in the ON state. UPDRS-III - Unified Parkinson's Disease Rating Scale Part III; MoCA - Montreal Cognitive Assessment; RBD-SQ - REM Sleep Behavior Disorder Screening Questionnaire; NMSQ - Non-motor Symptoms Questionnaire.

aggregates, in the form of Lewy bodies, spread across the brain in multiple stages affecting other structures, including locus coeruleus (LC), before the SNc. ${ }^{4,5}$ Despite insights gained through postmortem study of PD neuropathology, there is a lack of reliable biomarkers that can predict, diagnose, and track progression in PD. Neuroprotection trial

designs could be improved with brain imaging markers of neurodegeneration, which might assist participant selection and serve as surrogate outcome measures. Therefore, dependable and reproducible neuroimaging biomarkers are needed to detect and quantify PD neuropathology in vivo.

Melanized neurons in SNc and LC can be visualized in vivo with neuromelanin-sensitive MRI (NM-MRI) sequences using either incidental magnetization transfer effects from an interleaved multi-slice turbo spin echo acquisition ${ }^{6}$ or explicit magnetization transfer effects generated by magnetization transfer preparation pulses, ${ }^{7-9}$ Magnetization transfer contrast (MTC) colocalizes with melanized neurons in LC and SNc. ${ }^{10,11}$ NM-MRI approaches using incidental ${ }^{12}$ or explicit ${ }^{13-15}$ magnetization transfer effects have been found to exhibit high scan-rescan reproducibility in controls and gradient echo-based approaches with explicit magnetization transfer effects exhibit the highest reproducibility. ${ }^{15}$

NM-MRI can be used to assess PD-related reductions in tissue neuromelanin content in SNc and LC. Application of NM-MRI approaches based on incidental magnetization transfer effects to image depigmentation in SNc and LC has revealed $\mathrm{PD}$-related reductions in NM-MRI contrast ratios in SNc or LC, $, 16,17$ nigral volume, ${ }^{18}$ or area of SNc in a single slice. ${ }^{19,} 20$ Similar reductions have been observed in nigral volume $^{7}$ and contrast. ${ }^{21}$ Nigral regions of interest, derived from NM-MRI images, have also been used to examine PDrelated microstructural changes ${ }^{22,23}$ or iron deposition ${ }^{24,25}$ in SNc.
Replication of imaging markers in multiple cohorts is a crucial step in biomarker development and reliable detection of neuromelanin loss in SNc and LC may provide diagnostic imaging markers for PD. Reductions in nigral volume have been replicated in separate cohorts using explicit magnetization transfer effect-based NM-MRI approaches. ${ }^{26}$ However, PD-related reductions in LC volume loss have not been replicated and this deficiency may be due to the size and stature of LC, a small rod-shaped structure approximately $2 \mathrm{~mm}$ in diameter and $15 \mathrm{~mm}$ long. ${ }^{27}$ Here, we aim to remedy this deficiency and apply a NM-MRI approach based on explicit magnetization transfer effects to measure volume loss in LC as well as in SNc in PD patients and controls in separate discovery and validation cohorts acquired on different MRI scanner models.

\section{Methods}

\subsection{Research Participants}

Two cohorts, discovery and validation, were used in this study and were scanned on two different MRI scanners as described below. Cohort 1 data was collected from 20122014 and included 19 controls and 22 PD patients. Cohort 2 consisted of 33 healthy controls (HC) and 39 PD patients with data collected from 2015-2016. All research participants were recruited from the Emory University Movement Disorders Clinic under an institutional review board approved protocol with informed written consent. Controls were recruited from the community and the Emory Alzheimer's Disease Research Center control population. PD patients fulfilled the Movement Disorders Society clinical diagnostic criteria, ${ }^{28}$ and diagnosis was established by a fellowship-trained movement disorders neurologist at the Emory Movement Disorders Clinic. PD participants had early to moderate disease with Unified Parkinson's Disease 

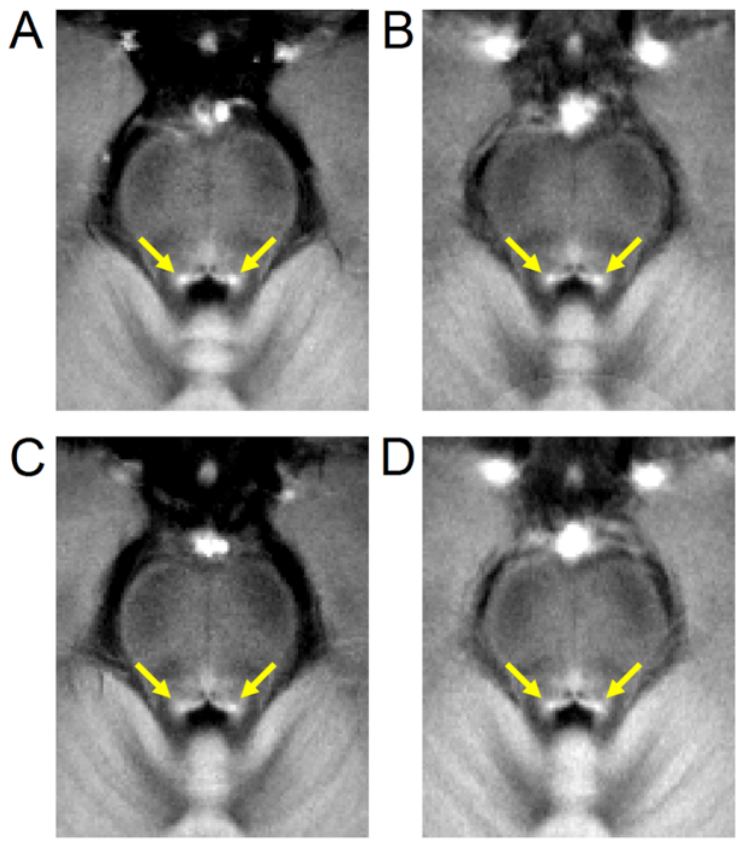

Figure 1. A comparison of mean LC contrast in control (top row) and PD (bottom row) groups for both cohorts. Mean MTC images from cohort 1 are shown in the left column while mean MTC images from cohort 2 are shown in the right column. For each group, the mean MTC image was created by transforming MTC images from individual participants to MNI space and then averaging. In each image, yellow arrows indicate the location of LC.

Rating Scale Part III (UPDRS-III) motor score $\leq 25$ in the practically defined ON state. Disease duration in years and levodopa equivalent daily dose (LEDD) were also determined for PD participants. Cognition was assessed using the Montreal Cognitive Assessment (MoCA). ${ }^{29}$ The Non-motor Symptoms Questionnaire (NMSQ) ${ }^{30}$ was used to assess non-motor symptoms. Symptoms of rapid eye

movement (REM) behavior disorder were assessed using the REM Sleep Behavior Disorder Screening Questionnaire (RBD-SQ). ${ }^{31}$

\subsection{MRI Acquisition}

MRI data for Cohort 1 were acquired with a Siemens Trio 3 Tesla scanner (Siemens Medical Solutions, Malvern, PA, USA) at Emory University with a 12-channel receiveonly head coil. NM-MRI data was acquired using a 2D magnetization transfer (MT) prepared gradient echo (GRE) sequence $^{8,}$ : : echo time $(\mathrm{TE}) /$ repetition time $(\mathrm{TR})=2.68 \mathrm{~ms}$ / $337 \mathrm{~ms}$, slice thickness $3 \mathrm{~mm}$, in plane resolution $0.39 \times 0.39$ $\mathrm{mm}^{2}$, field of view $(\mathrm{FOV})=162 \mathrm{~mm} \times 200 \mathrm{~mm}$, flip angle $(\mathrm{FA})=40^{\circ}, 470 \mathrm{~Hz} /$ pixel bandwidth, 15 contiguous slices, and MTC preparation pulse $\left(300^{\circ}, 1.2 \mathrm{kHz}\right.$ off resonance, 10 ms duration), 7 measurements, scan time 16 minutes 17 seconds. For registration from subject space to common space, $\mathrm{T}_{1}$ magnetization-prepared rapid gradient echo (MPRAGE) sequence was acquired with the following parameters: $\mathrm{TE} / \mathrm{TR}=3.02 \mathrm{~ms} / 2600 \mathrm{~ms}$, inversion time $=800$ $\mathrm{ms}, \mathrm{FA}=8^{\circ}$, voxel size $=1.0 \times 1.0 \times 1.0 \mathrm{~mm}^{3}$.

Cohort 2 was scanned with a Siemens Prisma 3 Tesla scanner using a 64-channel receive-only coil. NM-MRI data were acquired using a 2D GRE sequence with a MT preparation pulse: $\mathrm{TE} / \mathrm{TR}=3.10 \mathrm{~ms} / 354 \mathrm{~ms}, 15$ contiguous slices, $\mathrm{FOV}=162 \times 200 \mathrm{~mm}^{2}$, in plane resolution $=0.39 \times$ $\left.0.39 \times \mathrm{mm}^{2}\right), 7$ measurements, FA $=40^{\circ}, 470 \mathrm{~Hz} /$ pixel receiver bandwidth, and MTC pulses $\left(300^{\circ}, 1.2 \mathrm{kHz}\right.$ off resonance, $10 \mathrm{~ms}$ duration), scan time 17 minutes 12 seconds. For registration, structural images were acquired with a MP-RAGE sequence: TE/TR $=2.46 \mathrm{~ms} / 1900 \mathrm{~ms}$, inversion time $=900 \mathrm{~ms}, \mathrm{FA}=9^{\circ}$, voxel size $=0.8 \times 0.8 \times 0.8$ $\mathrm{mm}^{3}$.

On the sagittal $T_{1}$ images for both cohorts, the NM-MRI scan slices were positioned perpendicular to the dorsal edge of the brainstem at midline along the fourth ventricle, starting from the lower pons (below the most caudal extent of LC), with slices covering $\mathrm{SNc}$ and $\mathrm{LC}$.

\subsection{Image Processing}

MRI data was processed using the FMRIB Software Library (FSL). A transformation was derived between each individual's $\mathrm{T}_{1}$-weighted image and $2 \mathrm{~mm}$ Montreal Neurological Institute (MNI) $\mathrm{T}_{1}$-space using FMRIB's Linear Image Registration Tool (FLIRT) and FMRIB's Nonlinear Image Registration Tool (FNIRT) in the FSL software package using the following steps. ${ }^{32,} 33$ The $\mathrm{T}_{1}$ weighted image was brain extracted using the brain extraction tool (BET). Next, an affine transform was used to align the brain extracted $\mathrm{T}_{1}$-weighted images with the MNI brain extracted image. Finally, a nonlinear transformation was used to generate a transformation from individual $\mathrm{T}_{1}$ weighted images to $\mathrm{T}_{1}$-weighted $\mathrm{MNI} \mathrm{T}_{1}$-space.

For each participant individual NM-MRI measurements were corrected for motion by registering the seven measurements to the first image using a rigid-body transform in FMRIB Linear Image Registration Tool (FLIRT) and then averaged. Next, a transform was derived between each individual's $\mathrm{T}_{1}$-weighted image and the averaged NM-MRI image with a boundary-based registration cost function.

$\mathrm{SNc}$ and LC volumes were segmented using an automated thresholding method. To ensure consistent placement of reference regions of interest (ROIs), a reference ROI in the cerebral peduncle was created using the MNI template and, for each subject, the cerebral peduncle ROI was transformed to individual NM-MRI images using transforms described in previous paragraphs. This process ensured that the reference ROI was placed in similar locations for each subject. The mean (denoted $\mu_{\text {ref}}$ ), and standard deviation $\left(\sigma_{\mathrm{ref}}\right)$ of the signal intensities were measured in the reference ROI. Next, $\mathrm{SNc}$ and LC were segmented using probabilistic standard space masks. ${ }^{34}$ These atlases were transformed from standard space to individual NM-MRI images, thresholded at a level of 5\%, and dilated. Voxels in SNc and LC ROIs with intensity $>\mu_{\text {ref }}+3.9 \sigma_{\text {ref }}$ and 

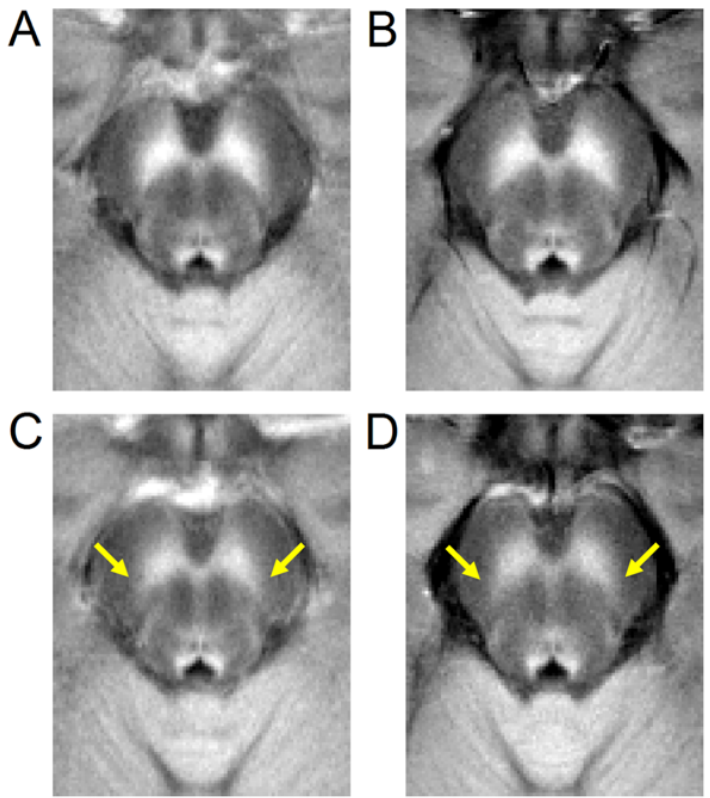

Figure 2. A comparison of mean SNc contrast in control (top row) and PD (bottom row) groups for both cohorts. Mean MTC images from cohort 1 are shown in the left column while mean MTC images from cohort 2 are shown in the right column. For each group, the mean MTC image was created by transforming MTC images from individual participants to MNI space and then averaging. Regions experiencing PD-related neuronal loss are indicated by yellow arrows in the bottom row.

$>\mu_{\text {ref }}+2.8 \sigma_{\text {ref }}$ were considered to be part of $\mathrm{LC}$ and $\mathrm{SNc}$, respectively.

\subsection{Statistical Analysis}

All statistical analyses were performed using IBM SPSS Statistics software version 24 (IBM Corporation, Somers, NY, USA) and results are reported as mean \pm standard error. A $p$ value of 0.05 was considered significant for all statistical tests performed in this work. Normality of $\mathrm{SNc}$ and $\mathrm{LC}$ volumes was assessed using the Shapiro-Wilk test for each group and all data was found to be normal.

For demographic data, independent samples t-test was used to assess differences in age and education and Chi square was used to examine differences in gender and race between controls and PD in each cohort. Group means for UPDRS-III score, disease duration, levodopa equivalents, MoCA, NMSQ, and RBD-SQ were compared using a twotailed Welch's $t$-test in each cohort.

In cohort 1, the effect of group (PD, control) was tested with separate analysis of covariance (ANCOVA) for $\mathrm{SNc}$ and LC volume controlling for age and education. In cohort 2, differences in $\mathrm{SNc}$ volume and $\mathrm{LC}$ volume between control and PD groups were compared using a twotailed Welch's t-test. Pearson's correlation was used to assess the relationship between SNc volume with UPDRSIII, LEDD, MoCA and LC volume in PD for cohort 1, cohort 2, and both cohorts combined. Pearson's correlation was used to assess the relationship between LC volume with UPDRS-III and MoCA for cohort 1, cohort 2, and both cohorts combined. Age was a treated as a covariate in all correlations.

\section{Results}

In cohort 1 , significant differences were in age $\left(p<10^{-3}\right)$ and education $(p=0.015)$ with controls being older and having higher levels of education than the PD group. No differences were seen between groups in gender $(p=0.453)$ or in MoCA score $(p=0.556)$. The PD group had significantly higher UPDRS-III $\left(p<10^{-3}\right)$, RBD-SQ $(p=0.002)$, and NMSQ $\left(p<10^{-3}\right)$ scores compared to the control group. In cohort 2, no difference was seen between PD and control groups in age $(p=0.894)$, race $(p=0.402)$, gender $(p=0.500)$, education $(p=0.661)$, RBD-SQ score $(p=0.087)$, or MoCA score $(p=0.621)$. A significant difference was seen in NMSQ $(p=0.0006)$ and UPDRS-III $\left(p<10^{-3}\right)$ scores between the two groups. Demographic information is summarized in Table 1.

Figures 1 and 2 show LC and SNc in mean MTC images of both cohorts. In cohort 1 , the effect of group on volume was assessed with separate ANCOVAs for each ROI (SNc, LC) with age and education as covariates. In $\mathrm{SNc}$, results revealed a significantly smaller volume in the PD group relative to the control group (Control: $474 \mathrm{~mm}^{3} \pm 31 \mathrm{~mm}^{3}$; PD: $\left.340 \mathrm{~mm}^{3} \pm 28 \mathrm{~mm}^{3} ; F=8.031 ; p=0.007\right)$. Similarly, a reduction in LC volume was seen in the PD group relative to the control group (Control: $6.9 \mathrm{~mm}^{3} \pm 0.7 \mathrm{~mm}^{3}$; PD: $4.4 \mathrm{~mm}^{3}$ $\pm 0.7 \mathrm{~mm}^{3} ; F=8.031 ; p=0.033$ ). In cohort 2 , Welch's $t$-test was used to examine group differences in SNc volume and LC volume. SNc (Control: $429 \mathrm{~mm}^{3} \pm 20 \mathrm{~mm}^{3}$; PD: 329 $\left.\mathrm{mm}^{3} \pm 17 \mathrm{~mm}^{3} ; t=3.370 ; p=0.0002\right)$ and LC (Control: 8.0 $\mathrm{mm}^{3} \pm 0.6 \mathrm{~mm}^{3}$; PD: $5.2 \mathrm{~mm}^{3} \pm 0.6 \mathrm{~mm}^{3} ; t=3.306$; $p=0.0008$ ) volumes were significantly lower in the $\mathrm{PD}$ group compared to the control group in cohort 2. These comparisons are shown in Figure 3.

In the PD group, MoCA showed a significant positive association with SNc volume in cohort $1(p=0.012, r=0.478)$ and both cohorts $(p=0.040, r=0.228)$ but not in cohort 2 $(p=0.319, r=0.079)$. SNc volume showed no significant associations with UPDRS-III or disease duration in the PD group in cohort 1 , cohort 2 or both cohorts ( $p s>0.436)$. There were no significant correlations between MoCA, UPDRS-III
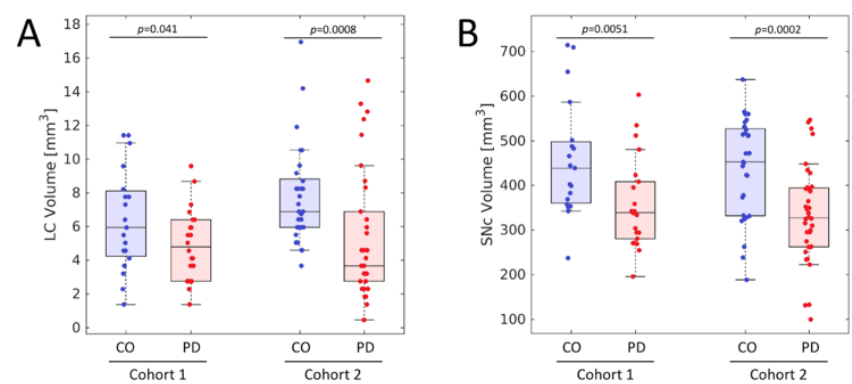

Figure 3. Group comparisons for LC volume (A) and SNc volume (B) in both cohorts. Similar reductions in $\mathrm{LC}$ and $\mathrm{SNc}$ volume are observed in both cohorts. In (A) and (B), the box denotes the $25^{\text {th }}$ and $75^{\text {th }}$ percentile, respectively, with the line denoting the median value. 


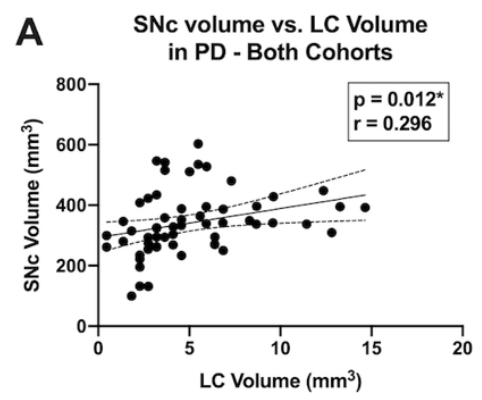

and disease duration with LC volume in cohort 1 , cohort 2, or both cohorts combined for the PD group $(p s>0.272)$. SNc volume showed a significant positive association with LC volume in PD in both cohorts $(p=0.012, r=0.296)$ but no significance in $\mathrm{HC}(p=0.434, r=-0.025)$. In the PD group, $\mathrm{SNc}$ and LC volumes had a significant positive correlation in cohort $2(p=0.024, r=0.333)$ but not in cohort $1 \quad(p=0.138$, $r=0.243)$. These correlations are shown in Figure 4.

In cohort $1 \mathrm{SNc}$ volume outperformed LC volume as a diagnostic imaging marker of $\mathrm{PD}$. The area under the

receiver operating characteristic (ROC) curve (AUC) for SNc volume was 0.756 [standard error (SE): 0.078, 95\% confidence interval (CI): $0.603-0.909, p=0.005]$ while the AUC for LC volume was 0.644 [SE: $0.088,95 \%$ CI: $0.471-$ $0.816, p=0.117]$. SNc volume and LC volume performed similarly in cohort 2 as diagnostic imaging markers of PD. LC volume had an AUC of 0.752 [SE: 0.063 , 95\% CI: $0.629-$ $0.876, p=0.001]$ and SNc volume had an AUC of 0.749 [SE: 0.062 , 95\% CI: $0.627-0.871, p=0.001]$.

\section{Discussion}

This study examined PD-related loss of NM-MRI contrast in LC and SNc in separate discovery and validation cohorts, using two different scanner models. We observed significant volume loss in both LC and SNc in the PD groups as compared to controls in both cohorts. In cohort $2, \mathrm{SNc}$ volume and LC volume performed similarly as diagnostic imaging markers of PD. This is the first report, to our knowledge, of reproducible detection of PD-associated LC volume loss using the same NM-MRI approach in multiple cohorts. It is also the first report of simultaneous imaging of $\mathrm{LC}$ and SNc detecting PD effects in both structures in discovery and validation cohorts. The NM-MRI pulse sequence and image processing methods that were used in this study have previously established high scan-rescan reproducibility. ${ }^{13,}{ }^{15}$ To increase reproducibility and ensure consistent placement of ROIs, reference regions and thresholded regions were defined using standard space ROIs and transformed to each individual's NM-MRI image. These ROIs were then used to define thresholded and reference regions in the $\mathrm{SNc}$ and $\mathrm{LC}$ segmentation procedure. The results of this study, therefore, support the utility of these NM-MRI methods as a robust and reproducible approach to measure PD neuropathology in vivo.

The two Parkinsonian cohorts used in this study had similar motor impairment, but different disease durations. The mean and standard error of nigral volume was strikingly
Figure 4. $\mathrm{SNc}$ volume vs. $\mathrm{LC}$ volume in $\mathrm{PD}$ (shown in A) and $\mathrm{HC}$ (shown in B) in both cohorts. SNc volume was positively correlated with LC volume in PD but not in $\mathrm{HC}$ in both cohorts.

consistent across both cohorts. Similarly, no difference was observed in LC volume between cohorts ( $p=0.081$, nonsignificant). The consistency in SNc and LC volume loss may be due to similarity of NM-MRI protocols used in this study. This is in contrast to an earlier study which found LC volume to be dependent on scanner, ${ }^{26}$ which used different implementations of NM-MRI protocols to image LC and the variability in scanning protocols may explain the differences in LC volume.

The ROC analysis found AUC of nigral volume in both cohorts and LC volume in cohort 2 to have comparable AUCs to previously published nigral diagnostic imaging markers of PD. ${ }^{21}, 26,35-38$ However, the performance of cohort's 1 LC volume as a diagnostic marker of PD was significantly below AUCs reported in earlier studies. ${ }^{21,26,35-38}$ Reduced performance of LC volume in cohort 1 may be due to several factors. First, the Siemens Trio MRI scanner used in cohort 1 has a higher noise profile than the Siemens Prisma MRI scanner used in cohort 2 and elevated noise likely reduced efficacy of LC volume as a diagnostic marker in cohort 1 . Second, the control group was significantly older than the PD group in cohort 1. Neuromelanin peaks in LC between age 50-60 and declines after age $60 .{ }^{39-41}$ Thus, this age difference likely reduced the effect size by reducing the mean LC volume in the control group.

Nigrosome-1 is the subregion of SNc that experiences the greatest reductions in melanized neurons ${ }^{42}$ and is found in the lateral-ventral portion of SNc. ${ }^{1}$ This region can be visualized in $\mathrm{T}_{2}$ - or $\mathrm{T}_{2} *$-weighted images as a hyperintense region in the substantia nigra and the loss of hyperintensity of nigrosome- 1 in $\mathrm{T}_{2}$ - or $\mathrm{T}_{2}{ }^{*}$-weighted images of $\mathrm{PD}$ patients is used as a diagnostic imaging marker. ${ }^{43}$ In NM-MRI images, nigrosome-1 has been localized to the posterior portion of SNc. ${ }^{44}$ We observed reductions in NM-sensitive contrast in the posterior regions of SNc (see Figure 2) in both cohorts. This is in agreement with earlier studies that found volume loss or reductions in neuromelanin-sensitive contrast in the posterior portion of $\mathrm{SNc}^{45}$ or in the lateral-ventral tier of SNc. ${ }^{21}$ Studies examining PD-related changes using other MRI contrasts have found increases in free water, ${ }^{46,} 47$ associated with neuronal loss, in posterior nigral ROIs as well as elevated iron levels in the lateral-ventral tier. ${ }^{48,} 49$ Taken together, these changes may manifest from neurodegeneration in nigrosome-1.

Animal studies using 1-methyl-4-phenyl-1,2,3,6tetrahydropyridine (MPTP) to induce Parkinsonism in mouse and primate models found that lesioning LC increases the 
medRxiv preprint doi: https://doi.org/10.1101/2022.02.23.22271356; this version posted February 24, 2022. The copyright holder for this

preprint (which was not certified by peer review) is the author/funder, who has granted medRxiv a license to display the preprint in perpetuity.

All rights reserved. No reuse allowed without permission.

amount of dopaminergic neurons lost in $\mathrm{SNc}^{50-53}$ and histology in humans found elevated levels of dopamine loss in PD correlates with reduced noradrenaline. ${ }^{54}$ Thus, integrity of LC neurons may protect dopaminergic neurons in SNc. In cohort 2, SNc and LC volume were significantly correlated in the PD group and this result may indicate that LC integrity plays a key role in Parkinsonian neurodegeneration in SNc. However, given the small sample size, different disease durations between cohorts, and the correlation not being replicated in cohort 1, these results should be interpreted with caution.

There are several caveats to this study. First, UPDRS-III was measured in the on-medication state. Earlier studies found a significant relationship between nigral volume and UPDRS score $^{26}$ and lack of correlation between nigral volume and UPDRS-III score may be due to measurement in the on medication state or lack of inclusion of PD patients with more severe motor symptoms. Second, the control group in cohort 1 was significantly older and more educated than the PD group. This may have reduced the effect size for LC volume.

\section{Conclusion}

NM-MRI approaches based on explicit magnetization transfer effects have already demonstrated high scan-rescan reproducibility. ${ }^{13-15}$ The current findings provide additional evidence that NM-MRI robustly detects PD-related reductions in volume in both $\mathrm{LC}$ and SNc. Therefore, further investigation of these SNc and LC volumes from NM-MRI as candidate PD biomarkers is warranted, both individually and as part of multivariate marker profiles using ironsensitive and diffusion-sensitive MRI modalities.

\section{Acknowledgements}

This work was supported by 1K23NS105944-01A1 (Huddleston) from the National Institutes of Health/National Institute of Neurological Diseases and Stroke, Michael J. Fox Foundation grants (MJFF-010556 and MJFF-010854), and the American Parkinson's Disease Foundation Center for Advanced Research at Emory University (Huddleston).

\section{References}

1. Fearnley JM, Lees AJ. Ageing and Parkinson's disease: substantia nigra regional selectivity. Brain 1991;114 ( Pt 5):2283-2301.

2. Dauer W, Przedborski S. Parkinson's disease: mechanisms and models. Neuron 2003;39(6):889-909.

3. Cheng HC, Ulane CM, Burke RE. Clinical progression in Parkinson disease and the neurobiology of axons. Ann Neurol 2010;67(6):715-725.

4. Braak H, Del Tredici K, Rub U, de Vos RA, Jansen Steur EN, Braak E. Staging of brain pathology related to sporadic Parkinson's disease. Neurobiol Aging 2003;24(2):197-211.
5. Braak H, Ghebremedhin E, Rub U, Bratzke H, Del Tredici K. Stages in the development of Parkinson's disease-related pathology. Cell Tissue Res 2004;318(1):121-134.

6. Sasaki M, Shibata E, Tohyama K, et al. Neuromelanin magnetic resonance imaging of locus ceruleus and substantia nigra in Parkinson's disease. Neuroreport 2006;17(11):1215-1218.

7. Ogisu K, Kudo K, Sasaki M, et al. 3D neuromelanin-sensitive magnetic resonance imaging with semi-automated volume measurement of the substantia nigra pars compacta for diagnosis of Parkinson's disease. Neuroradiology 2013;55(6):719-724.

8. Chen X, Huddleston DE, Langley J, et al. Simultaneous imaging of locus coeruleus and substantia nigra with a quantitative neuromelanin MRI approach. Magn Reson Imaging 2014;32(10):1301-1306.

9. Langley J, Huddleston DE, Chen X, Sedlacik J, Zachariah N, Hu $\mathrm{X}$. A multicontrast approach for comprehensive imaging of substantia nigra. Neuroimage 2015;112:7-13.

10. Keren NI, Taheri S, Vazey EM, et al. Histologic validation of locus coeruleus MRI contrast in post-mortem tissue. Neuroimage 2015;113(1):235-245.

11. Kitao S, Matsusue E, Fujii S, et al. Correlation between pathology and neuromelanin MR imaging in Parkinson's disease and dementia with Lewy bodies. Neuroradiology 2013;55(8):947953.

12. Tona $\mathrm{KD}$, Keuken $\mathrm{MC}$, de Rover $\mathrm{M}$, et al. In vivo visualization of the locus coeruleus in humans: quantifying the test-retest reliability. Brain Struct Funct 2017.

13. Langley J, Huddleston DE, Liu CJ, Hu X. Reproducibility of locus coeruleus and substantia nigra imaging with neuromelanin sensitive MRI. MAGMA 2017;30(2):121-125.

14. Wengler K, He X, Abi-Dargham A, Horga G. Reproducibility assessment of neuromelanin-sensitive magnetic resonance imaging protocols for region-of-interest and voxelwise analyses. Neuroimage 2020;208:116457.

15. van der Pluijm M, Cassidy C, Zandstra M, et al. Reliability and Reproducibility of Neuromelanin-Sensitive Imaging of the Substantia Nigra: A Comparison of Three Different Sequences. J Magn Reson Imaging 2021;53(3):712-721.

16. Ohtsuka C, Sasaki M, Konno K, et al. Changes in substantia nigra and locus coeruleus in patients with early-stage Parkinson's disease using neuromelanin-sensitive MR imaging. Neurosci Lett 2013;541:93-98

17. Matsuura K, Maeda M, Yata K, et al. Neuromelanin magnetic resonance imaging in Parkinson's disease and multiple system atrophy. Eur Neurol 2013;70(1-2):70-77.

18. Castellanos G, Fernandez-Seara MA, Lorenzo-Betancor O, et al. Automated Neuromelanin Imaging as a Diagnostic Biomarker for Parkinson's Disease. Mov Disord 2015;30(7):945-952.

19. Schwarz ST, Rittman T, Gontu V, Morgan PS, Bajaj N, Auer DP. T1-weighted MRI shows stage-dependent substantia nigra signal loss in Parkinson's disease. Mov Disord 2011;26(9):16331638.

20. Reimao S, Pita Lobo P, Neutel D, et al. Substantia nigra neuromelanin magnetic resonance imaging in de novo Parkinson's disease patients. Eur J Neurol 2015;22(3):540-546. 
medRxiv preprint doi: https://doi.org/10.1101/2022.02.23.22271356; this version posted February 24, 2022. The copyright holder for this preprint (which was not certified by peer review) is the author/funder, who has granted medRxiv a license to display the preprint in perpetuity.

All rights reserved. No reuse allowed without permission.

21. Huddleston DE, Langley J, Sedlacik J, Boelmans K, Factor SA, $\mathrm{Hu}$ XP. In vivo detection of lateral-ventral tier nigral degeneration in Parkinson's disease. Hum Brain Mapp 2017;38(5):2627-2634.

22. Pyatigorskaya N, Magnin B, Mongin M, et al. Comparative Study of MRI Biomarkers in the Substantia Nigra to Discriminate Idiopathic Parkinson Disease. AJNR Am J Neuroradiol 2018;39(8):1460-1467.

23. Langley J, Huddleston DE, Merritt M, et al. Diffusion tensor imaging of the substantia nigra in Parkinson's disease revisited. Hum Brain Mapp 2016;37(7):2547-2556.

24. Isaias IU, Trujillo P, Summers P, et al. Neuromelanin Imaging and Dopaminergic Loss in Parkinson's Disease. Front Aging Neurosci 2016;8:196.

25. Langley J, He N, Huddleston DE, et al. Reproducible detection of nigral iron deposition in 2 Parkinson's disease cohorts. Mov Disord 2019;34(3):416-419.

26. Schwarz ST, Xing Y, Tomar P, Bajaj N, Auer DP. In Vivo Assessment of Brainstem Depigmentation in Parkinson Disease: Potential as a Severity Marker for Multicenter Studies. Radiology 2016:160662.

27. Sasaki M, Shibata E, Tohyama K, et al. Monoamine neurons in the human brain stem: anatomy, magnetic resonance imaging findings, and clinical implications. Neuroreport 2008;19(17):16491654.

28. Postuma RB, Berg D, Stern M, et al. MDS clinical diagnostic criteria for Parkinson's disease. Mov Disord 2015;30(12):15911601.

29. Nasreddine ZS, Phillips NA, Bedirian V, et al. The Montreal Cognitive Assessment, MoCA: a brief screening tool for mild cognitive impairment. J Am Geriatr Soc 2005;53(4):695-699.

30. Chaudhuri KR, Martinez-Martin P, Schapira AH, et al. International multicenter pilot study of the first comprehensive selfcompleted nonmotor symptoms questionnaire for Parkinson's disease: the NMSQuest study. Mov Disord 2006;21(7):916-923.

31. Stiasny-Kolster K, Mayer G, Schafer S, Moller JC, HeinzelGutenbrunner M, Oertel WH. The REM sleep behavior disorder screening questionnaire--a new diagnostic instrument. Mov Disord 2007;22(16):2386-2393.

32. Smith SM, Jenkinson M, Woolrich MW, et al. Advances in functional and structural MR image analysis and implementation as FSL. NeuroImage 2004;23 Suppl 1:S208-219.

33. Woolrich MW, Jbabdi S, Patenaude B, et al. Bayesian analysis of neuroimaging data in FSL. NeuroImage 2009;45(1 Suppl):S173186.

34. Langley J, Hussain S, Flores JJ, Bennett IJ, Hu X. Characterization of age-related microstructural changes in locus coeruleus and substantia nigra pars compacta. Neurobiol Aging 2020;87:89-97.

35. Cheng Z, He N, Huang P, et al. Imaging the Nigrosome 1 in the substantia nigra using susceptibility weighted imaging and quantitative susceptibility mapping: An application to Parkinson's disease. Neuroimage Clin 2020;25:102103.

36. Li G, Zhai G, Zhao X, et al. 3D texture analyses within the substantia nigra of Parkinson's disease patients on quantitative susceptibility maps and R2( *) maps. Neuroimage 2019;188:465472.

37. Ariz M, Abad RC, Castellanos G, et al. Dynamic Atlas-Based Segmentation and Quantification of Neuromelanin-Rich Brainstem Structures in Parkinson Disease. IEEE Trans Med Imaging 2019;38(3):813-823.

38. Shinde S, Prasad S, Saboo Y, et al. Predictive markers for Parkinson's disease using deep neural nets on neuromelanin sensitive MRI. Neuroimage Clin 2019;22:101748.

39. Ma SY, Roytt M, Collan Y, Rinne JO. Unbiased morphometrical measurements show loss of pigmented nigral neurones with ageing. Neuropathol Appl Neurobiol 1999;25(5):394-399.

40. Zecca L, Stroppolo A, Gatti A, et al. The role of iron and copper molecules in the neuronal vulnerability of locus coeruleus and substantia nigra during aging. Proc Natl Acad Sci U S A 2004;101(26):9843-9848.

41. Manaye KF, McIntire DD, Mann DM, German DC. Locus coeruleus cell loss in the aging human brain: a non-random process. J Comp Neurol 1995;358(1):79-87.

42. Damier P, Hirsch EC, Agid Y, Graybiel AM. The substantia nigra of the human brain. II. Patterns of loss of dopaminecontaining neurons in Parkinson's disease. Brain 1999;122 ( Pt 8):1437-1448

43. Blazejewska AI, Schwarz ST, Pitiot A, et al. Visualization of nigrosome 1 and its loss in PD: pathoanatomical correlation and in vivo 7 T MRI. Neurology 2013;81(6):534-540.

44. Langley J, Huddleston DE, Crosson B, Song DD, Factor SA, Hu $\mathrm{X}$. Multimodal assessment of nigrosomal degeneration in Parkinson's disease. Parkinsonism \& related disorders 2020;80:102107.

45. Schwarz ST, Rittman T, Gontu V, Morgan PS, Bajaj N, Auer DP. T1-Weighted MRI shows stage-dependent substantia nigra signal loss in Parkinson's disease. Mov Disord 2011;26(9):16331638 .

46. Planetta PJ, Ofori E, Pasternak O, et al. Free-water imaging in Parkinson's disease and atypical parkinsonism. Brain 2016;139(Pt 2):495-508.

47. Zhou L, Li G, Zhang Y, et al. Increased free water in the substantia nigra in idiopathic REM sleep behaviour disorder. Brain 2021;144(5):1488-1497.

48. He N, Langley J, Huddleston DE, et al. Increased irondeposition in lateral-ventral substantia nigra pars compacta: A promising neuroimaging marker for Parkinson's disease. Neuroimage Clin 2020;28:102391.

49. Langley J, Huddleston DE, Sedlacik J, Boelmans K, Hu XP. Parkinson's disease-related increase of T2*-weighted hypointensity in substantia nigra pars compacta. Mov Disord 2017;32(3):441-449.

50. Bing G, Zhang Y, Watanabe Y, McEwen BS, Stone EA. Locus coeruleus lesions potentiate neurotoxic effects of MPTP in dopaminergic neurons of the substantia nigra. Brain Res 1994;668(1-2):261-265.

51. Fornai F, Alessandri MG, Torracca MT, Bassi L, Corsini GU. Effects of noradrenergic lesions on MPTP/MPP+ kinetics and 
medRxiv preprint doi: https://doi.org/10.1101/2022.02.23.22271356; this version posted February 24, 2022. The copyright holder for this preprint (which was not certified by peer review) is the author/funder, who has granted medRxiv a license to display the preprint in perpetuity.

All rights reserved. No reuse allowed without permission.

MPTP-induced nigrostriatal dopamine depletions. J Pharmacol Exp Ther 1997;283(1):100-107.

52. Yao N, Wu Y, Zhou Y, et al. Lesion of the locus coeruleus aggravates dopaminergic neuron degeneration by modulating microglial function in mouse models of Parkinsons disease. Brain Res 2015;1625:255-274.

53. Rommelfanger KS, Weinshenker D, Miller GW. Reduced MPTP toxicity in noradrenaline transporter knockout mice. J Neurochem 2004;91(5):1116-1124.

54. Tong J, Hornykiewicz O, Kish SJ. Inverse relationship between brain noradrenaline level and dopamine loss in Parkinson disease: a possible neuroprotective role for noradrenaline. Arch Neurol 2006;63(12):1724-1728. 